\title{
Comparison of Antenna Radiation Efficiency Measurement Techniques in Reverberation Chamber Using or Not a Reference Antenna
}

\author{
W. Krouka ${ }^{1}$, F. Sarrazin ${ }^{1}$, J. Sol ${ }^{2}$, P. Besnier ${ }^{2}$, E. Richalot ${ }^{1}$ \\ ${ }^{1}$ Univ Paris-Est, ESYCOM FRE 2028, CNAM, CNRS, ESIEE Paris, F-77454, Marne-la-vallée, France, \\ ${ }^{2}$ Univ Rennes, INSA Rennes, CNRS, IETR - UMR 6164, F-35000 Rennes, France \\ ${ }^{1}$ wafa.krouka@u-pem.fr \\ ${ }^{2}$ philippe.besnier@insa-rennes.fr
}

\begin{abstract}
Antenna characterization using reverberation chamber (RC) has become the new trend in RC measurements. In this work, we compare three antenna characterization methods in RC. These methods are applied in order to determine the radiation efficiency of a patch and a log-periodic antenna. Results are compared and show good coherence. The accuracy of the applied methods is discussed and a solution is proposed in order to enhance the accuracy of reference antenna based methods.
\end{abstract}

\section{INTRODUCTION}

In addition to electromagnetic compatibility measurements, RCs are being lately used for antenna measurements. Especially in order to evaluate the antenna radiation efficiency. This is a relevant measurement for general purpose or miniature antennas not dedicated to reach a dedicated antenna pattern requirement.

A well performing $\mathrm{RC}$ presents a statistically isotropic and homogenous electric field within the RC working volume with the help of different stirring techniques (mechanical, source, frequency...) [1]. This allows conducting accurate antenna measurements, in particular in order to retrieve global parameters such as the antenna radiation efficiency [2], [3]. Such properties are usually obtained for measurements at frequencies much higher than the RC lower usable frequency (LUF).

The very first proposed methods for antenna characterization in RCs were based on the use of a reference antenna $\left(\operatorname{Ref}_{\text {ant }}\right)$ with known efficiency, in addition to the antenna under test (AUT) [4], [5]. However, author of reference [3] proposes new methods using one, two or three antennas in RC without the need of any $\mathrm{Ref}_{\text {ant }}$. This is an important breakthrough since a $\operatorname{Ref}_{\text {ant }}$ is hardly available. Most selected reference antennas are wide band antennas with supposedly constant efficiency (e.g. $90 \%$ for a $\log$ periodic antenna) in its whole frequency range. In realistic case, this hypothesis considering a fixed value of $\operatorname{Ref}_{\text {ant }}$ radiation efficiency over its entire frequency band is not very accurate [4]. Thus, we need to first have a better estimation of the Ref $_{\text {ant }}$ radiation efficiency in order to enhance the measurement accuracy of the AUT radiation efficiency.

In section II, we present the three methods that have been applied in order to determine both patch and log-periodic antenna radiation efficiencies in IETR's RC. Section III is dedicated to the presentation of the measurement setup and processing.

In section IV, efficiency measurement results are presented and compared. A better estimation of $\operatorname{Ref}_{\text {ant }}$ radiation efficiency is proposed and better efficiency results of the AUTs are presented and compared with results obtained by the classical method. Finally, section $\mathrm{V}$ gives the conclusion for this work.

\section{MEASUREMENT METHODS}

Three different methods are applied in order to determine the radiation efficiency of a patch and a logperiodic antenna. Two of them are $\mathrm{Ref}_{\text {ant }}$ based methods and will be noted respectively as "method (a)" and "method (b)" in the following sections. The third method does not require the use of a $\operatorname{Ref}_{\text {ant }}$ and will be noted as "method (c)".

\section{A. Measurement method (a)}

This method is one of the classical methods used for antenna radiation efficiency measurement. It consists of the substitution of the AUT for a $\operatorname{Ref}_{\text {ant }}$. Thus, measurements are conducted in two steps. First, a transmit antenna is used with the AUT, then, keeping the same transmit antenna, the AUT is substituted for the $\operatorname{Ref}_{\text {ant }}$. This method is based on the hypothesis that $\operatorname{Ref}_{\text {ant }}$ with known radiation efficiency has a fixed value over the entire measurement frequency band. The AUT radiation efficiency $\left(\eta_{A U T}\right)$ can be expressed as a function of the $\operatorname{Ref}_{\text {ant }}$ radiation efficiency $\left(\eta_{\text {Ref }_{\text {ant }}}\right)[5]$

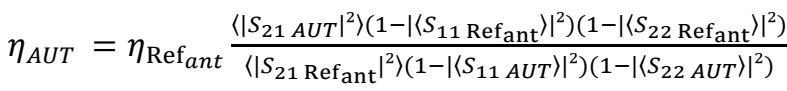

where: $\langle.\rangle_{\theta}$ refers to the average over all stirrer positions $\theta$. $S_{i j A U T}, S_{i j \operatorname{Ref}_{\text {ant }}}$ are the scattering parameters associated to the AUT and the $\operatorname{Ref}_{\text {ant }}$, respectively. Eq. 1 is based on the assumption of having the same calculated quality factor during the first measurement with AUT and the second one where $\operatorname{Ref}_{\text {ant }}$ is used. 


\section{B. Measurement method (b)}

Two antennas are used in this method including the

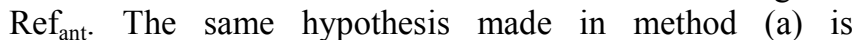
considered here. Therefore, radiation efficiency of the $\operatorname{Ref}_{\text {ant }}$ is estimated to have fixed value over the entire measurement frequency band. The composite quality factor " $Q$ "of the RC can be expressed as [5]

$$
Q_{i}=\left\langle\left|S_{i i, s}\right|^{2}\right\rangle \frac{z_{0} \omega \varepsilon . V}{\left(\lambda^{2} / 4 \pi\right)\left(1-\left|\left\langle S_{i i}\right\rangle\right|^{2}\right)^{2} \eta_{i}^{2}}
$$

with $Z_{0}$ : free space impedance, $\omega=2 . \pi$. $f$ with $f$ : frequency, $\varepsilon$ : dielectric permittivity, $\mathrm{V}: \mathrm{RC}$ volume, $\eta_{i}$ : radiation efficiency of antenna " $i$ ", $S_{i i, s}:$ stirred component of $S_{i i}$ parameter defined as

$$
S_{i i, s}=S_{i i}-\left\langle S_{i i}\right\rangle_{\theta}
$$

Reflection coefficients of both antennas can be used in eq. 2 in order to determine the composite quality factor. We can then write: $Q_{1}=Q_{2}$. If we consider that antenna 1 is the reference antenna with known efficiency $\eta_{1}$ then, we can write: $\eta_{2}=\sqrt{\frac{Q_{2}^{\prime}}{Q_{1}}}$

$$
\text { and: } \quad Q_{2}^{\prime}=\left\langle\left|S_{22, S}\right|^{2}\right\rangle \frac{Z_{0} \omega \varepsilon . V}{\left(\lambda^{2} / 4 \pi\right)\left(1-\left|\left\langle S_{22}\right\rangle\right|^{2}\right)^{2}}
$$

\section{Measurement method (c)}

This method does not require a $\operatorname{Ref}_{\text {ant }}$ and it is based on the difference between the time domain estimation of the $\mathrm{RC}$ quality factor and the frequency domain estimation of the latter. In the hypothesis of negligible losses at antenna ports with regard to wall losses, the time domain estimation of the quality factor is slightly higher than its frequency domain estimation due to the non-ideality of antennas. Two important parameters must be determined: the $\mathrm{RC}$ time constant $\tau_{R C}$ (proportional to the RC quality factor) and the enhanced backscattered coefficient $e b$ (a figure of merit of field homogeneity inside the RC). Two measurement antennas are used. $\tau_{R C}$ is defined as the inverse of the slope calculated from the logarithmic representation of power delay profile $P D P(t)$. The latter is expressed as a function of the RC transfer function as

$$
P D P(t)=\left\langle\left|I F T\left[S_{21}\right]\right|^{2}\right\rangle_{\theta}=P_{0} \exp \left(-t / \tau_{R C}\right)
$$

where IFT is the inverse Fourier transform, $S_{21}$ is the transmission coefficient between the two antennas and $\langle.\rangle_{\theta}$ is the average over all stirrer positions $\theta$. The hypothesis made by this method is that the $Q$ factor (here evaluated from the decay time estimation) represents the composite quality factor of the $\mathrm{RC}$ in the hypothesis of negligible losses introduced by antennas with regard to other losses in the chamber.

Once we have calculated $\tau_{R C}, Q$ can be determined as [3] $e b$ is expressed as

$$
Q=\omega \tau_{R C}
$$

$$
e b=\frac{\sqrt{\left\langle\left|S_{11, s}\right|^{2}\right\rangle\left\langle\left|S_{22, s}\right|^{2}\right\rangle}}{\left\langle\left|S_{21, s}\right|^{2}\right\rangle}
$$

We may then demonstrate (see details in [3]), that antenna radiation efficiency of measurement antennas (or AUT) is represented as

$$
\eta_{i}=\sqrt{\frac{\left\langle\left|S_{i i, s}\right|^{2}\right\rangle \cdot C_{R C}}{\left(1-\left|\left\langle S_{i i}\right\rangle\right|^{2}\right)^{2} \cdot Q \cdot e b}}
$$

with: $C_{R C}=\frac{16 \pi^{2} V}{\lambda^{3}}$

\section{MEASUREMENT SETUP AND PROCESSING}

Measurements have been conducted in IETR RC (Fig. 1). The chamber is equipped with a metallic stirrer and has a total volume of $93.35 \mathrm{~m}^{3}$.

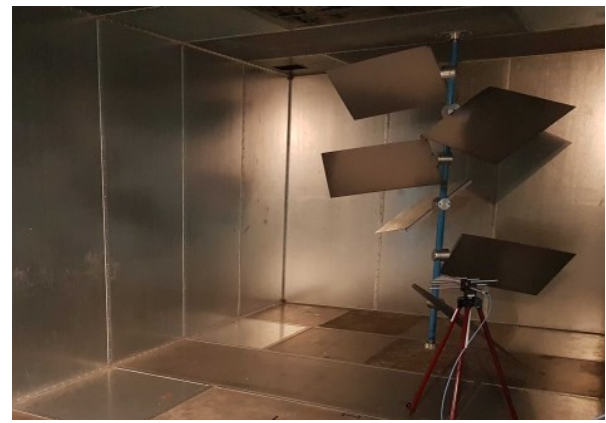

Fig. 1. RC of IETR laboratory.

The LUF of IETR RC is estimated around $200 \mathrm{MHz}$. The frequency band considered for measurements is chosen well above the LUF: [1.2 GHZ - 3.2 GHz]; Antenna losses are therefore negligible. Antennas that have been characterized are presented in Fig. 2.

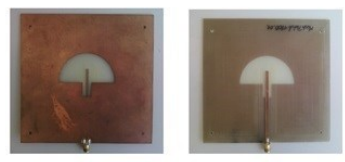

Patch antenna

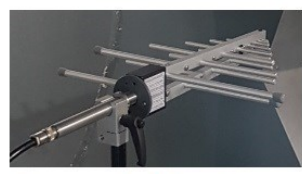

Log-periodic antenna
Fig. 2. Antennas to be characterized.

Both antennas in Fig. 2 are used in method (b) and (c) whereas a third one is used as a transmit antenna in method c. The latter is a horn antenna with a usable frequency band of $[750 \mathrm{MHz}-18 \mathrm{GHz}]$. The patch antenna is printed on an FR4 substrate and it is made of a half-disc slot excited by a monopole. This antenna is broadband and matched between 1.75 and $2.7 \mathrm{GHz}\left(S_{11}\right.$ below $\left.-6 \mathrm{~dB}\right)$.

The log periodic antenna is broadband and has a usable frequency band of [ $340 \mathrm{MHz}-4 \mathrm{GHz}]$. 
This antenna was used as $\operatorname{Ref}_{\text {ant }}$ in both method (a) and method (b) detailed previously. Measurements were performed in the RC using an Agilent Technologies $20 \mathrm{GHz}$ VNA over 10.001 equally spaced frequency samples. The stirrer was rotated with a $3.6^{\circ}$ angular step thus providing 100 measurements over a complete rotation of the stirrer.

The measurement setup is presented in Fig. 3. As far as the methods (b) and (c) are concerned, antennas (1) and (2) are sketched in black color depicting that the patch antenna and the log periodic antenna are used simultaneously. On the other hand, in method (a), the used antennas are represented in grey color. A first measurement is conducted using antenna (2) and (3) (antenna (3) represents the transmit antenna) and in a second measurement, antenna (1) is substituted for antenna (2) while keeping the same transmit antenna (3).

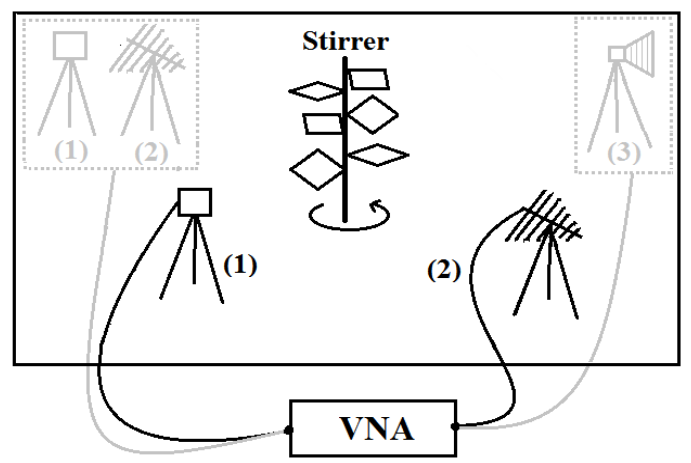

Fig. 3. Measurement Setup.

Depending on the selected method, different post processing is applied.

- $\quad$ Method (a) and method (b)

Average values are calculated using measurements collected over the 100 stirrer positions. For a better presentation of the radiation efficiency results, the obtained result is smoothed over a sliding frequency window of $30 \mathrm{MHz}$. Radiation efficiency of the reference antenna is considered constant and equal to $90 \%$ over the entire frequency band (hypothesis made by both methods).

\section{- $\quad$ Method (c)}

Average values are calculated using measurements collected over the 100 stirrer positions. The RC time constant is calculated over sliding window of $100 \mathrm{MHz}$ and the result is associated to the central frequency of each window. The obtained result is smoothed over a sliding frequency window of $30 \mathrm{MHz}$.

In order to verify the hypothesis made by method (c), we calculate the quality factor of both antennas. In Fig. 4, we present quality factor of patch and $\log$ periodic $\left(\mathrm{Ref}_{\mathrm{ant}}\right)$ antennas compared to the time domain quality factor of RC. We notice that $\mathrm{Q}$ factors of both antennas are very high compared to the calculated $\mathrm{Q}$ factor. Therefore, we can consider that antennas have a small contribution in the composite Q factor. These results confirm that the hypothesis made by method (c) is verified.

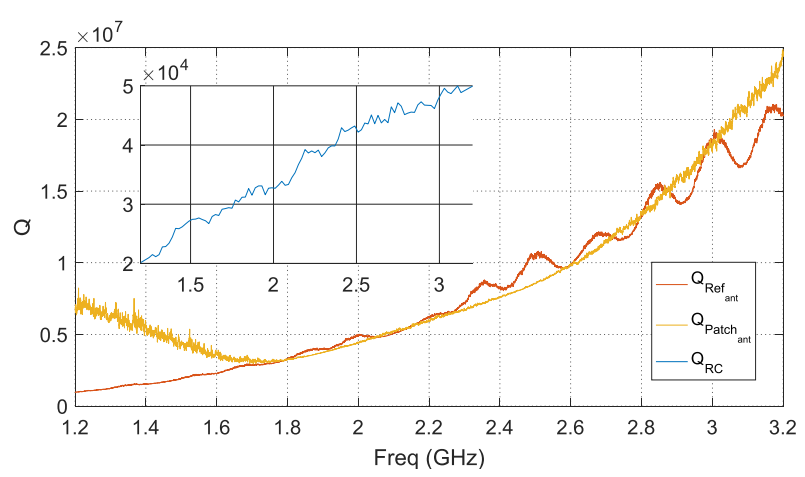

Fig. 4. RC and antennas quality factors.

\section{MEASUREMENT RESULTS}

In this section we present radiation efficiency results obtained for patch and log periodic antenna $\left(\operatorname{Ref}_{\text {ant }}\right)$ using the three methods presented in section II. Fig. 5 presents very coherent results for the three methods. We notice that starting from frequency $2.1 \mathrm{GHz}$, result obtained using method (c) presents lower values compared to results of method (a) and (b). At $3.2 \mathrm{GHz}$ the difference reaches $6 \%$. The same behavior is noticed for radiation efficiency results of the log periodic antenna $\left(\operatorname{Ref}_{\text {ant }}\right)$ in Fig. 6 when compared to the hypothetically fixed value of $90 \%$.

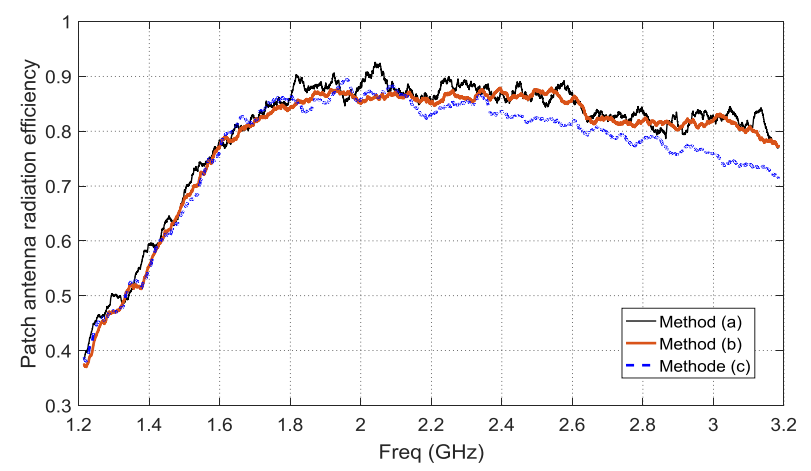

Fig. 5. Patch antenna radiation efficiency using the three methods versus frequency.

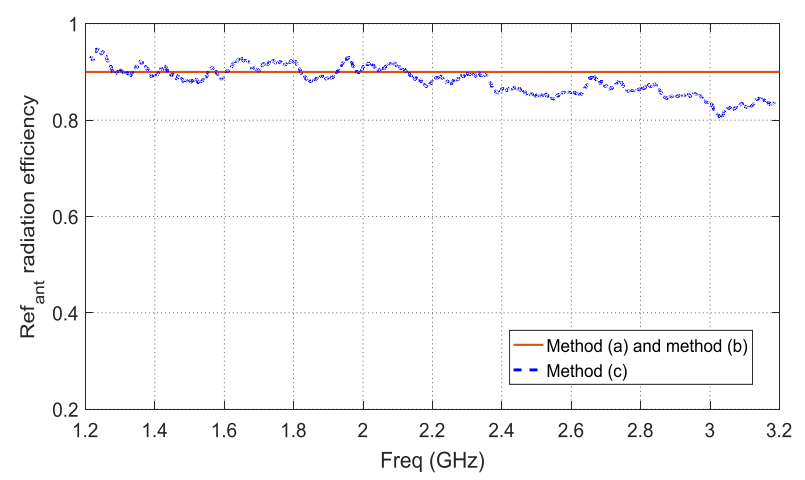

Fig. 6. Log periodic $\left(\operatorname{Ref}_{a n t}\right)$ radiation efficiency. Its value is supposed fixed and equal to $90 \%$ for methods (a) and (b) whereas it is measured using method (c). 
These results show that the hypothesis taking a fixed value for $\mathrm{Ref}_{\text {ant }}$ radiation efficiency might not be validated, according to the value estimated by method (c). The estimation error of $\mathrm{Ref}_{\text {ant }}$ radiation efficiency led to nonaccurate estimation of the patch antenna radiation efficiency. In order to overcome this problem, we suggest a prior evaluation of the $\operatorname{Ref}_{\text {ant }}$ radiation efficiency (with a characterization method that does not require a $\left.\operatorname{Ref}_{\mathrm{ant}}\right)$ in order to have a real estimation of its value. Radiation efficiency of $\operatorname{Ref}_{\text {ant }}$ obtained using method (c) can then be used in methods (a) and (b).

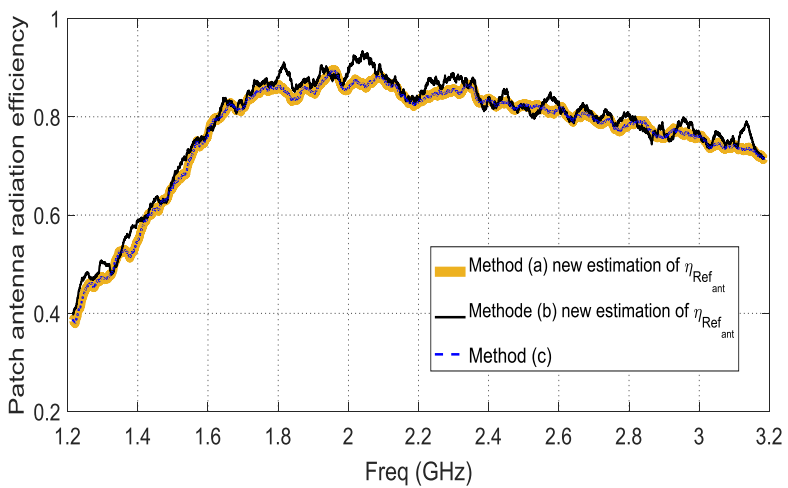

Fig. 7. Patch antenna radiation efficiency with the new estimation of $\operatorname{Ref}_{\text {ant }}$ radiation efficiency.

Fig. 7 shows very similar results obtained with the three methods (a), (b) and (c).

The new estimation of $\mathrm{Ref}_{\text {ant }}$ radiation efficiency using method (c) allows us to have a better estimation of the patch antenna radiation efficiency.

\section{CONCLUSIONS}

This paper presents results for antenna radiation efficiency measurements obtained using three different methods: substitution method (noted method (a)), quality factor method (noted method (b)) and non-reference antenna method (noted method (c)) in RC. Unlike method (c), methods (a) and (b) are based on the use of a reference antenna with a known radiation efficiency that is hypothetically constant over the entire measurement frequency band.

Using method (c), we have determined the radiation efficiency of a patch and a $\log$ periodic antenna. The latter has then been used as a $\operatorname{Ref}_{\text {ant }}$ in methods (a) and (b). Over more than half of measurement frequency band, radiation efficiency of the log periodic antenna does not present stable values (around $90 \%$ ) as it has been considered in methods (a) and (b). This estimation error if not corrected generates inaccurate results for the AUT radiation efficiency with methods (a) and (b). This has been noticed on the patch antenna radiation efficiency results. In order to overcome this estimation error, we propose a prior determination of the $\mathrm{Ref}_{\text {ant }}$ radiation efficiency (for reference antenna-based methods) in order to have accurate results for AUTs radiation efficiency.

\section{ACKNOWLEDGMENT}

This project was supported in part by the European Union through the European Regional Development Fund, in part by the Ministry of Higher Education and Research, in part by the Région Bretagne, and in part by the Département d'Ille et Vilaine and Rennes Métropole, through the CPER Project SOPHIE/STIC \& Ondes.

\section{REFERENCES}

[1] R. Serra et al., "Reverberation chambers a la carte: An overview of the different mode-stirring techniques," Electromagn. Compat. Mag., vol. 6, no. 1, pp. 63-78, Jan.-Mar. 2017.

[2] W. Krouka, F. Sarrazin, E.Richalot, "Influence of the Reverberation Chamber on Antenna Characterization Performances", International Symposium on Electromagnetic Compatibility - EMC EUROPE, 2018.

[3] Christopher L.Holloway, Haider A.Shah, Ryan J.Pirkl, William F.Young, David A.Hill and John Ladbury, "Reverberation chamber techniques for determining the radiation and total efficiency of antennas". IEEE transactions on antennas and propagation, Vol .60, No 4, April, 2012.

[4] P. Hallbjorner, Reflective antenna efficiency measurements in reverberation chambers, Microwave Opt. Tecnol. Lett, vol. 30, no. 5, pp 332-335, Sept 2001.

[5] P. Besnier, J. Sol, A. Presse, C. Lemoine and A. C. Tarot, "Antenna efficiency measurement from quality factor estimation in reverberation chamber," European Microwave Conference (EuMC), London, pp. 715-718, 2016 